\title{
Simple LED spectrophotometer for analysis of color information
}

\author{
Ji-Sun Kim, A-Hee Kim, Han-Byeol Oh, Bong-Jun Goh, Eun-Suk Lee, Jun-Sik Kim, Gu-In \\ Jung, Jin-Young Baek and Jae-Hoon Jun* \\ Department of Biomedical Engineering, BK21 Plus Research Institute of Biomedical Engineering, \\ College of Biomedical and Health Science, Konkuk University, Chungju, Republic of Korea
}

\begin{abstract}
A spectrophotometer is the basic measuring equipment essential to most research activity fields requiring samples to be measured, such as physics, biotechnology and food engineering. This paper proposes a system that is able to detect sample concentration and color information by using LED and color sensor. Purity and wavelength information can be detected by CIE diagram, and the concentration can be estimated with purity information. This method is more economical and efficient than existing spectrophotometry, and can also be used by ordinary persons. This contribution is applicable to a number of fields because it can be used as a colorimeter to detect the wavelength and purity of samples.
\end{abstract}

Keywords: Color information, color space, light emitting diode, spectrophotometer

\section{Introduction}

Due to recent advances in science and technology, the detection of specimen color or wavelength using a spectrophotometer, colorimeter, or turbidimeter is frequently used in most laboratories and hospitals [1-4]. The color measuring tool is the basic measuring equipment essential to most research activity fields, such as physics, biotechnology and food engineering [5-9]. The soluble solid content, oil content, acidity, and other physiological properties of various fruits and vegetables have been detected in food industry [10]. Cell viability was tested with a colorimetric method using a reductive coloring reagent such as tetrazoliumsalt, alamarBlue and PrestoBlue in biotechnology [11, 12]. Spectrometry can be used in the analysis of pollutants in water and the ocean by measuring the Chemical Oxygen Demand (COD) of organic matter using hydrogen peroxide $\left(\mathrm{H}_{2} \mathrm{O}_{2}\right)$ and potassium permanganate $\left(\mathrm{KMnO}_{4}\right)[13,14]$. Spectrophotometry has also been used to evaluate interactions between antimicrobial membrane peptides and lipid bilayers [15]. Other optical methods to detect bioparticles have reported with the use of a microfluidic device and UV absorbance [16].

A spectrophotometer sorts incident light by wavelength and compares the brightness of light [17]. However, spectrophotometry has disadvantages in that the equipment is expensive and large, and an expert is required to analyze the resulting data. Additionally, a spectrophotometer cannot verify

\footnotetext{
* Address for correspondence: Jae-Hoon Jun, Department of Biomedical Engineering, College of Biomedical and Health Science, Konkuk University, 268 Chungwondaero, Chungju, Chungbuk, 380-701, Republic of Korea. Tel.: +82 438403799 ; Fax: +82 43 8454814; E-mail: jjun81@kku.ac.kr.
} 
measurement errors and is not suitable for an experiment that continuously measures the change of signal over time $[18,19]$. As a method to replace the function of spectrophotometer, a colorimeter can measure the absorption of specific wavelengths of light by comparing a sample with the absorption of a standard color. However, this method requires information on the standard color to be measured, is highly affected by the external environment, and demonstrates low resolution [20]. As the importance of spectrophotometry increases, research on miniature spectrophotometers has been conducted, but miniature spectrophotometry has several disadvantages: it must reduce the number of optical filters, and is sensitive to interference, causing performance to deteriorate [21, 22]. As described above, most color information is obtained by using spectrophotometry and colorimeter, but is limited for use by non-professionals. Therefore, this paper investigated how to obtain optical color information of a sample using photodiode. A system that can quantify optical information (e.g., wavelength, purity) of a sample was built. Results indicate that the proposed system can detect discoloration and even slight differences in color among samples. Because color information can be obtained only in the presence of a light source and photodiode, the system can be designed for small volume samples due to the simplicity of the setup as compared to traditional spectrophotometry. This system is a non-contact measurement method and can obtain color information without contamination or loss of sample volume. This study applies to fields requiring the measurement of physiological properties of fruit or meat, the testing of cell viability, and the detection of pollutants or particles.

\section{Materials and methods}

\subsection{CIE diagram}

Figure 1 shows the wavelength region of visible light detected by photoreceptor cells. The CIE (Commission Internationale de l'Eclairage) diagram is a standard colorimetric system based on the wavelength region shown in Figure 1 and generally referred to as the XYZ colorimetric system. The standard chromaticity determined by this method is referred to as CIE 1931 xy chromaticity. CIE 1931 is primarily used for scientific technology and in the creation of dye or the mixing of paints.

Figure 2 shows the CIE chromaticity diagram used in this study. An important color space, defined by the Commission Internationale de l'Eclairage, is designated as the CIE XYZ color space. RGB values were obtained with an array of the color sensor using a LabVIEW computer program. These values were input into the mathematical formula to obtain coordinate color values in CIE color space. Subsequently, the distance from the coordinate value to a spectrum line could ultimately detect the wavelength and purity value of a color. For example, the purity value of the coordinate situated at the spectrum line in the color space is $100 \%$. As the point $\mathrm{A}$ is close to point $\mathrm{E}$ (white), the purity value is decreased and can be calculated as the ratio of EA/EB [23].
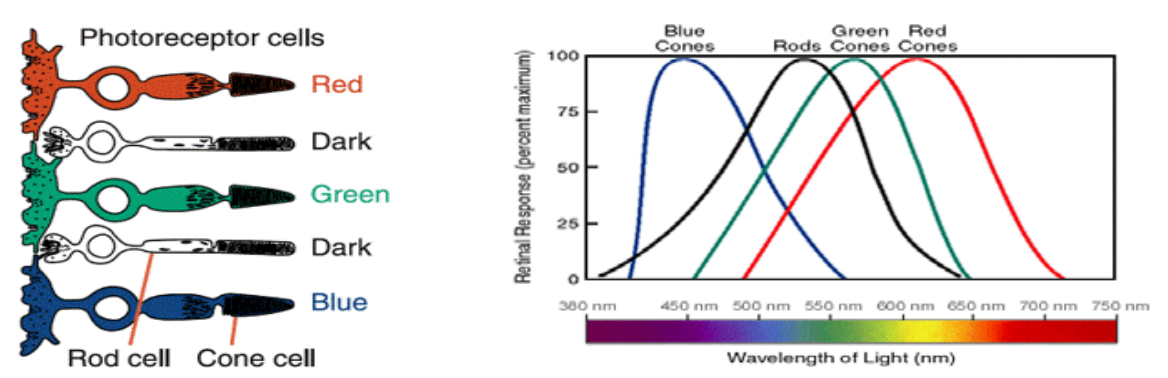

Fig. 1. Spectral sensitivity of various photoreceptors. 


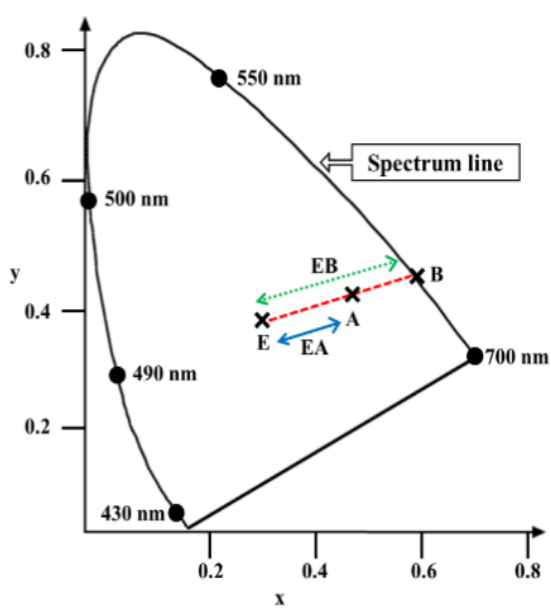

Fig. 2. CIE chromaticity diagram.

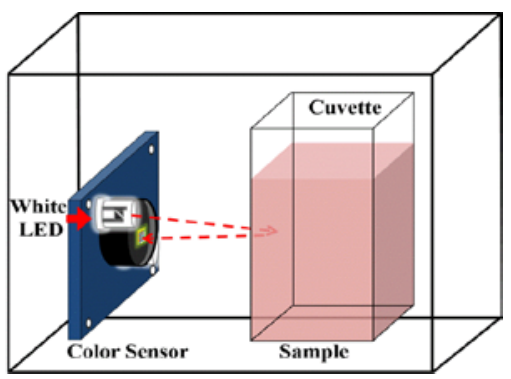

Fig. 3. Sensor setup.

Conversion of RGB to XYZ takes the form of a simple matrix transformation. Chromaticity coordinates $\mathrm{x}$ and $\mathrm{y}$ are obtained from the values $\mathrm{X}, \mathrm{Y}$, and $\mathrm{Z}$ as described in Eq. (2). The CIE XYZ color space was deliberately designed so that the $\mathrm{Y}$ parameter was a measure of the brightness or luminance of a color. The chromaticity of a color can then be specified by the two derived parameters $\mathrm{x}$ and $\mathrm{y}$, two of the three normalized values which are functions of all three tristimulus values $\mathrm{X}, \mathrm{Y}$, and $\mathrm{Z}[24]$.

$$
\begin{gathered}
X=0.412 * R+0.358 * G+0.180 * B \\
Y=0.213 * R+0.715 * G+0.072 * B \\
Z=0.019 * R+0.119 * G+0.950 * B \\
\mathrm{x}=\mathrm{X} /(\mathrm{X}+\mathrm{Y}+\mathrm{Z}) \\
\mathrm{y}=\mathrm{Y} /(\mathrm{X}+\mathrm{Y}+\mathrm{Z})
\end{gathered}
$$

\subsection{Experimental design}

Figure 3 illustrates the experimental setup configuration. The experimental setup was composed of a white LED (Light-Emitting Diode), self-developed cuvette, and optical receiver. The white LED served as the light source, as LEDs offer more stability, reliability, longer life, and smaller size than other light sources. The light generated by LED is incident on the photodiode through the sample in an acrylic transparent cuvette. The produced sample concentrations (mass concentration $[\mathrm{g} / \mathrm{g}]$ ) ranged from 0.05 to $0.25 \%$. General water paint was used to create samples, and the information of the light incident on the photodiode was calculated with LabVIEW software and converted into color coordinates.

\subsection{Light detection module}

Figure 4 shows the structure of the color sensor and LED used in the experiment. In order to measure color information, a white LED was used as the light source. LEDs offer the advantages of low power consumption, a high efficient product and low cost. The color sensor used in the 


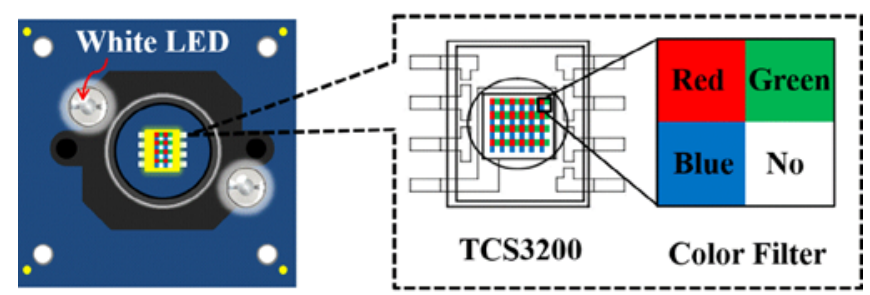

Color Sensor

Fig. 4. Structure of color sensor.

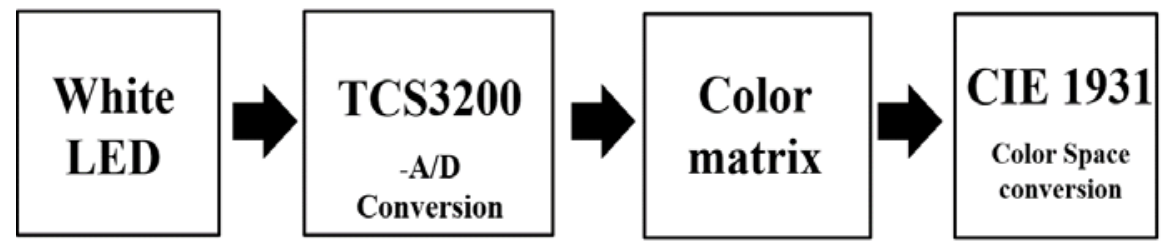

Fig. 5. Flow chart to convert RGB values into wavelength and purity.

experiment to configure the LED spectrophotometer was a TCS3200 (TAOS, USA) and converts the light signal into a digital value. In this sensor, photodiodes are arranged in an array form and optical filters (red, green, and blue) are attached to each photodiode. The passing ranges of the optical filters are $580 \mathrm{~nm}-680 \mathrm{~nm}, 450 \mathrm{~nm}-610 \mathrm{~nm}$, and $380 \mathrm{~nm}-580 \mathrm{~nm}$ for red, green, and blue filters, respectively. It is composed of 12 photodiodes; the light signal entered into a photodiode, and is converted into a digital signal. Next, the digital value output from the sensor shows a value between 0 and 255, the values representing black to white, respectively. The value and color coordinates can be calculated by applying these numerical values to Eqs. (1) and (2), and thus the wavelength and purity values can be detected.

Figure 5 illustrates the schematic diagram of the experimental procedures. The white LED produces light, and the color intensity is detected by the color sensor (TCS3200). Using chromaticity coordinates (see Eqs. (1) and (2)), the wavelength and purity can be obtained using LabVIEW computer software.

\section{Results and discussion}

Figure 6 demonstrates the results of connecting color coordinates of red, green, blue, yellow, and violet with the spectrum line the concentration of the sample is $0.25 \%$. Wavelength information was obtained from the spectrum line, and the purity value from the center was obtained. The wavelength values of red, green, blue, and yellow are $620 \mathrm{~nm}, 540 \mathrm{~nm}, 481 \mathrm{~nm}$, and $572 \mathrm{~nm}$, respectively. The wavelength value of purple could not be identified, because the purple line in the CIE diagram does not provide wavelength values. The measured results are consistent with the wavelength values of theoretical visible rays (red band at approximately $600 \mathrm{~nm}$, green band at approximately $500 \mathrm{~nm}$, and blue band at approximately $400 \mathrm{~nm}$ ). Results demonstrate that wavelength values can be obtained by marking various colors in color coordinates, thus replacing spectrophotometer functionality.

Table 1 illustrates the changes in wavelength and purity values according to changes in sample concentration. The movement of color coordinates was observed by changing the concentrations of five colors in five steps, and subsequently detecting wavelength and purity. Changes in color cannot be detected with the naked eye, but the analysis of the experimental data showed that color coordinates 


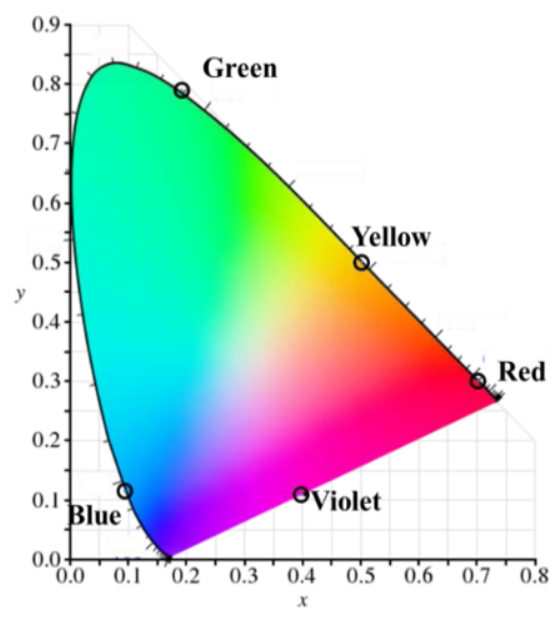

Fig. 6. Wavelength and purity changes in RGB values.

Table 1

Wavelength and purity changes according to changes in sample concentration

\begin{tabular}{|c|c|c|c|c|c|}
\hline Color & Concentration (\%) & $x$ & $y$ & Wavelength (nm) & Purity (\%) \\
\hline \multirow{5}{*}{ Red } & 0.05 & 0.54 & 0.31 & 628 & 56.41 \\
\hline & 0.1 & 0.56 & 0.31 & 623 & 62.71 \\
\hline & 0.15 & 0.57 & 0.31 & 622 & 64.99 \\
\hline & 0.2 & 0.57 & 0.32 & 621 & 66.94 \\
\hline & 0.25 & 0.58 & 0.32 & 620 & 70.08 \\
\hline \multirow{5}{*}{ Green } & 0.05 & 0.33 & 0.39 & 556 & 17.56 \\
\hline & 0.1 & 0.33 & 0.42 & 551 & 23.57 \\
\hline & 0.15 & 0.32 & 0.42 & 548 & 24.16 \\
\hline & 0.2 & 0.31 & 0.44 & 541 & 24.75 \\
\hline & 0.25 & 0.31 & 0.44 & 540 & 26.51 \\
\hline \multirow{5}{*}{ Blue } & 0.05 & 0.26 & 0.3 & 486 & 26.62 \\
\hline & 0.1 & 0.24 & 0.29 & 485 & 34.11 \\
\hline & 0.15 & 0.23 & 0.26 & 483 & 41.69 \\
\hline & 0.2 & 0.23 & 0.26 & 482 & 42.03 \\
\hline & 0.25 & 0.22 & 0.24 & 481 & 46.95 \\
\hline \multirow{5}{*}{ Violet } & 0.05 & 0.32 & 0.3 & - & 10.91 \\
\hline & 0.1 & 0.37 & 0.28 & - & 26.53 \\
\hline & 0.15 & 0.38 & 0.28 & - & 30.65 \\
\hline & 0.2 & 0.39 & 0.27 & - & 33.24 \\
\hline & 0.25 & 0.41 & 0.28 & - & 34.33 \\
\hline \multirow{5}{*}{ Yellow } & 0.05 & 0.38 & 0.38 & 578 & 26.19 \\
\hline & 0.1 & 0.38 & 0.38 & 577 & 29.11 \\
\hline & 0.15 & 0.38 & 0.39 & 576 & 31.23 \\
\hline & 0.2 & 0.38 & 0.4 & 574 & 33.00 \\
\hline & 0.25 & 0.38 & 0.41 & 572 & 38.53 \\
\hline
\end{tabular}


change with simultaneous changes in sample concentration. Additionally, as the sample concentration increased, purity values increased significantly. Thus, the results demonstrate that the concentration information of samples (mass concentration $[\mathrm{g} / \mathrm{g}]$ ) can be detected by purity values rather than wavelength values.

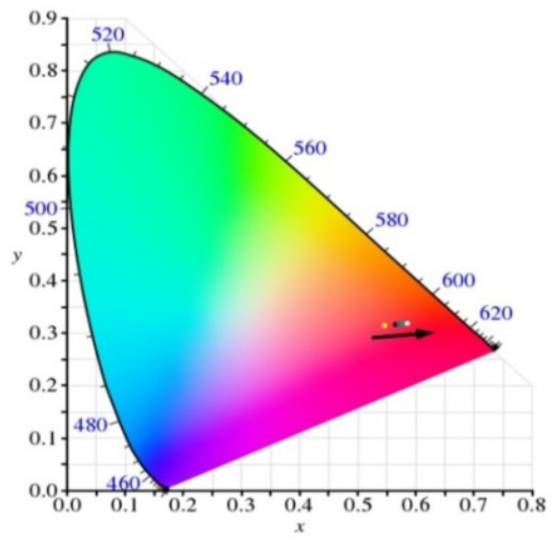

(a) Red

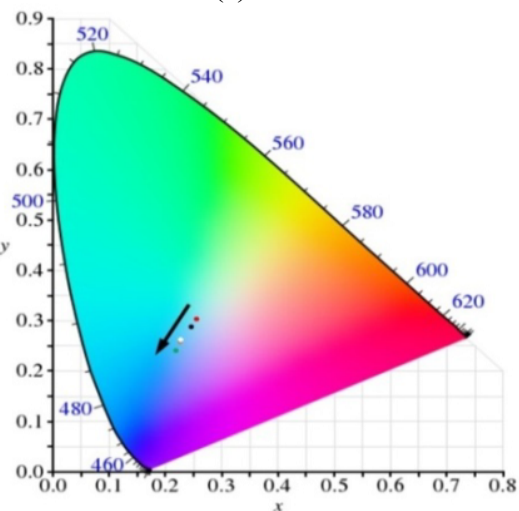

(c) Blue

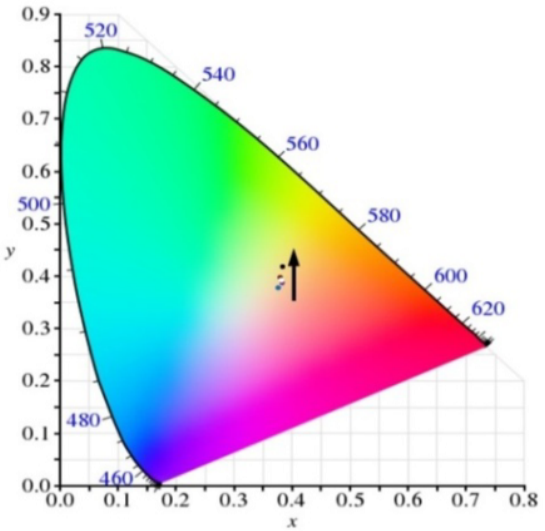

(e) Yellow

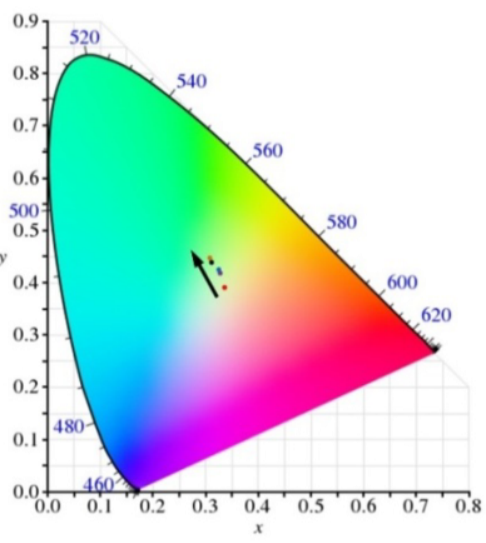

(b) Green

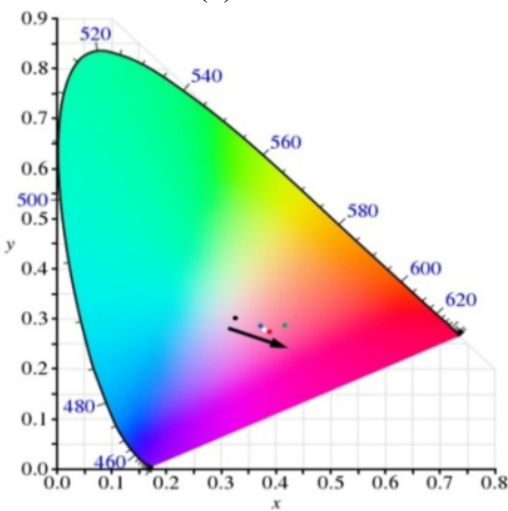

(d) Violet

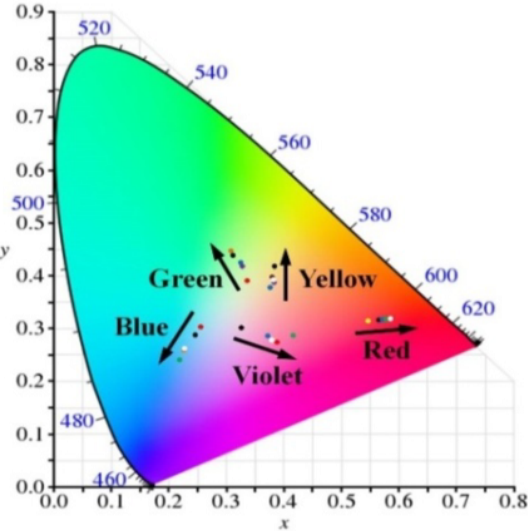

(f) All colors

Fig. 7. Color coordinate changes by RGB values. 
Figure 7 demonstrates the results of schematized color coordinates obtained by measuring RGB values in the sensor in the CIE diagram. Imaging data according to color coordinates has the advantages of stronger transmission effects and the use of more familiar information, which is useful in understanding the data. Additionally, it helps to easily identify trends that cannot be identified without visualization. In particular, as shown in Figure 7, it can be observed that as the concentration changes in various colors, color coordinates move toward the spectrum line (shown in Figure 2). This occurs because purity increases as sample concentration increases. Results indicate that trends can be examined and concentration of samples can be observed with color coordinates and purity values, as well as with wavelength as shown in the CIE diagram.

\section{Conclusion}

This study obtained RGB values of samples by using optical elements, and analyzed them according to color coordinates. In particular, RGB information of the sample was analyzed according to color coordinates, and wavelength values and purity values were obtained based on xy values. In addition, a portable system was implemented by creating a system with optical elements and miniaturizing the system, allowing ease of with appropriate software components. The proposed method can replace the function of the spectrophotometer, eliminating the need for expensive equipment. Additionally, data collected over time can be observed and saved using the LabVIEW computer software. The system can measure optical information of the samples regardless of sample type, thus utilizing a nondestructive examination method. This study presents a color information measuring system that can measure the state change and optical information of substances without being constrained by long analysis time and a specific location. These findings are beneficial because it can be used as colorimeter as well as collect absorbance and transmittance measurements.

\section{Acknowledgments}

This research was supported by Basic science Research Program through the National Research Foundation of Korea (NRF) funded by the Ministry of Education, science and Technology (No. 20100023158) and the Pioneer Research Center Program through the National Research Foundation of Korea funded by the Ministry of science, ICT \& Future planning (No. 2011-0027920).

\section{References}

[1] S. Nishino and K. Ohshima, A feasibility study of gender recognition with a near infrared ray scanning spectrophotometer, Electronics and Communications in Japan 94 (2011), 59-68.

[2] A.K. Gaigalas, L. Wang, V. Karpiak, Y.Z. Zhang and S. Choquette, Measurement of scattering cross section with a spectrophotometer with an integrating sphere detector, Journal of Research of the National Institute of Standards and Technology 117 (2012), 202-215.

[3] K.S. Johnson, L.J. Coletti, H.W. Jannasch, C.M. Sakamoto, D.D. Swift and S.C. Riser, Long-term nitrate measurements in the ocean using the in situ ultraviolet spectrophotometer: Sensor integration into the APEX profiling float, Journal of Atmospheric and Oceanic Technology 30 (2013), 1854-1866.

[4] A. Penzkofer, Optical rotatory dispersion measurement of D-glucose with fixed polarizer analyzer accessory in conventional spectrophotometer, Journal of Analytical Sciences, Method and Instrumentations 3 (2013), 234-239.

[5] K.Y. Khan, M.A. Khan, R. Niamat, M. Munir, H. Fazal, P. Mazari, N. Seema, T. Bashir, A. Kanwal and S. N. Ahmed, Element content analysis of plants of genus ficus using atomic absorption spectrometer, African Journal of Pharmacy and Pharmacology 5 (2011), 317-321.

[6] A. Burkart, S. Cogliati, A. Schickling and U. Rascher, A novel UAV-based ultra-light weight spectrometer for field spectroscopy, Sensors Journal, Institute of Electrical and Electronics Engineers 14 (2014), 62-67. 
[7] F. Antonucci, F. Pallottino, G. Paglia, A. Palma, S. D'Aquino and P. Menesatti, Non-destructive estimation of mandarin maturity status through portable VIS-NIR spectrophotometer, Food and Bioprocess Technology 4 (2011), 809-813.

[8] T. Atomssa and A.V. Gholap, Characterization of caffeine and determination of caffeine in tea leaves using Uv-visible spectrometer, African Journal of Pure and Applied Chemistry 5 (2011), 1-8.

[9] T. Makino and H. Wakabayashi, New spectrophotometer system for measuring thermal radiation characteristics of real surfaces of thermal engineering entirely, Journal of Thermal Science and Technology 6 (2011), 80-92.

[10] Y. Yagiz, M.O. Balaban, H.G. Kristinsson, B.A. Welt and M.R. Marshall, Comparison of minolta colorimeter and machine vision system in measuring colour of irradiated Atlantic salmon, Journal of the Science of Food and Agriculture 89 (2009), 728-730.

[11] M. Xu, D.J. McCanna and J.G. Sivak, Use of the viability reagent PrestoBlue in comparison with alamar blue and MTT to access the viability of human corneal epithelial cells, Journal of Pharmacological and Toxicological Methods 71 (2015), 1-7.

[12] S. Kolusheva, L. Boyer and R. Jelinek, A colorimetric assay for rapid screening of antimicrobial peptides, Nature Biotechnology 18 (2000), 225-227.

[13] N. Abdullah, H.A. Aziz, N.N.A.N. Yusuf, M. Umar and S.S.A. Amr, Potential of $\mathrm{KMnO}_{4}$ and $\mathrm{H}_{2} \mathrm{O}_{2}$ in treating semiaerobic landfill leachate, Applied Water Science 4 (2014), 303-309.

[14] A. Carabet, C. Florescu, C. Staniloju, C. Podoleanu, M. Visescu, M. Belu and C. Ilie, The efficiency of resita town water treatment plant, Revista de Chimie 64 (2013), 559-563.

[15] P. Kassal, R. Surina, D. Vrsaljko and I.M. Steinberg, Hydro sol-gel thin films doped with a pH indicator: Effect of organic modification on optical $\mathrm{Ph}$ response and film surface hydrophilicity, Journal of Sol-Gel Science and Technology 69 (2014), 586-595.

[16] M. Masrie, B.Y. Majlis and J. Yunas, Fabrication of multilayer-PDMS based microfluidic device for bio-particles concentration detection, Bio-Medical Materials and Engineering 24 (2014), 1951-1958.

[17] X. Gan, N. Pervez, I. Kymissis, F. Hatami and D. Englund, A high-resolution spectrometer based on a compact planar two dimensional photonic crystal cavity array, Applied Physics Letters 100 (2012), 231104.

[18] D.V. Spracklen, J.L. Jimenez, K.S. Carslaw, D.R. Worsnop, M.J. Evans, G.W. Mann, Q. Zhang, M.R. Canagaratna, J. Allan, H. Coe, G. McFiggans, A. Rap and P. Forster, Aerosol mass spectrometer constraint on the global secondary organic aerosol budget, Atmospheric Chemistry and Physics 11 (2011), 12109-12136.

[19] A. Wego, Accuracy simulation of an LED based spectrophotometer, Optik-International Journal for Light and Electron Optics 124 (2013), 644-649.

[20] T. Bano, G. Yadav and R. Dudhe, Development and validation of oseltamivir phosphate API by UV-spectrophotometer, Global Journal of Pharmacology 7 (2013), 294-297.

[21] C. Gomez-Polo, M. Gomez-Polo, A. Celemin-Vinuela and J.A.M.V. De Parga, Differences between the human eye and the spectrophotometer in the shade matching of tooth color, Journal of Dentistry 42 (2014), 742-745.

[22] T. Coin, S. Lee and S. Chin, Method of combining spectrophotometer and optical imaging equipment to extract optical parameters for material rendering, Journal of Sensors 2014 (2014), 1-8.

[23] L. Zhang, H. Kong, C.T. Chin, T. Wang and S. Chen, Cytoplasm segmentation on cervical cell images using graph cutbased approach, Bio-Medical Materials and Engineering 24 (2014), 1125-1131.

[24] X. Wang, Uniformity research on CIE 1931 chromaticity diagram, Commission Internationale de l'Eclairage 178 (2008), $1-84$. 